\title{
UTILIZAÇÃO DE ÓLEOS ESSENCIAIS NA CONSERVAÇÃO DE QUEIJO: REVISÃO
}

\section{Use of essential oils in cheese conservation: Review}

\author{
Maria Gleiciane Soares Coutinho ${ }^{I^{*}}$, Andréa Maria Neves ${ }^{2}$, Ana Sancha Malveira Batista ${ }^{3}$, \\ Raquel Oliveira dos Santos Fontenelle
}

\begin{abstract}
RESUMO
O queijo é um dos alimentos mais consumidos no Brasil, por exibir ampla aceitação em todas as classes sociais. Contudo, é bastante susceptível a contaminação microbiana. As doenças de origem alimentar, causadas principalmente por bactérias, são um sério problema de saúde pública, sendo a causa de várias mortes em todo o mundo. Diante do exposto, o presente estudo objetivou, a partir de uma revisão bibliográfica, discorrer sobre a conservação de queijos produzidos com óleos essenciais, de modo a incentivar que novas pesquisas sejam realizadas almejando descobrir compostos que possam ser utilizados na conservação de alimentos, principalmente queijos. Nesta revisão foram abordadas algumas variedades de queijos mais comercializados, citando suas características e forma de contaminação, principais micro-organismos associados à contaminação do queijo, tais como Listeria monocytogenes, Salmonella spp., Escherichia coli e Staphylococcus aureus, plantas com potencial antimicrobiano frente a bactérias envolvidas na contaminação do queijo, conservantes sintéticos e estudos relatando a inibição do crescimento de alguns micro-organismos em queijos produzidos com adição de óleos essenciais extraídos de plantas.
\end{abstract}

Palavras-chave: bactéria; intoxicação; antibacteriano; conservação.

1 Universidade Estadual do Ceará, Departamento de Ciências e Tecnologia, Avenida Dr. Silas Munguba, 1700, Itaperi, 60714-903, Fortaleza, CE, Brasil. E-mail: gleycy-soares1@hotmail.com

2 Universidade Estadual do Ceará, Departamento de Biotecnologia, Fortaleza, CE, Brasil

3 Universidade Estadual Vale do Acaraú, Departamento de Zootecnia, Sobral, CE, Brasil.

4 Universidade Estadual Vale do Acaraú, Departamento de Ciências Biológicas, Sobral, CE, Brasil.

* Autor para correspondência 


\begin{abstract}
Cheese is one of the most consumed foods in Brazil, as it shows wide acceptance in all social classes. However, it is highly susceptible to microbial contamination. Food-borne diseases, caused mainly by bacteria, are a serious public health problem and are the cause of several deaths worldwide. In view of the above, the present study aimed at a bibliographical review to discuss the conservation of cheese produced with essential oils, in order to encourage new research to be carried out aiming to discover compounds that can be used in food preservation, mainly cheeses. In this review, some varieties of more commercialized cheeses were mentioned, citing their characteristics and form of contamination, the main microorganisms associated with cheese contamination, such as Listeria monocytogenes, Salmonella spp., Escherichia coli, and Staphylococcus aureus, plants with antimicrobial potential against bacteria involved in cheese contamination, synthetic preservatives and studies reporting the inhibition of growth of some microorganisms in cheeses produced with the addition of essential oils extracted from plants.
\end{abstract}

Keywords: bacteria; intoxication; antibacterial; conservation.

\section{INTRODUÇÃO}

Um dos problemas de saúde pública mais frequentes atualmente são as doenças transmitidas por alimentos (DTA), que ocorrem pela ingestão de água e alimentos contaminados, principalmente por microorganismos, sendo as bactérias as maiores responsáveis pelos surtos e mortes de origem alimentar (FORSYTHE, 2013).

Os micro-organismos podem se associar aos alimentos em qualquer etapa do processo de produção, como manuseio, processamento, acondicionamento, estocagem, distribuição e/ ou no preparo para o consumo, gerando assim, a contaminação, permitindo que produtos de baixa qualidade cheguem aos consumidores. Os micro-organismos que sobreviverem aos processos aplicados durante o preparo e acondicionamento dos alimentos poderão causar intoxicação ao consumidor (FAVA et al., 2012).

O aumento dos casos de DTA contribui para o desenvolvimento de cepas com resistência, podendo trazer consequências graves a saúde pública, ao reduzir as alterna- tivas terapêuticas. Os alimentos contribuem para a disseminação de cepas patogênicas resistentes. Torna-se uma situação preocupante, pois, na produção animal, são utilizados vários antimicrobianos que desempenham pressão seletiva sobre os micro-organismos (CARDOSO; MARIN, 2014).

O queijo, por sua rica composição nutricional, torna-se bastante susceptível a proliferação microbiana, apresentando contaminações inclusive por microorganismos patogênicos, que podem ser potenciais causadores de doenças (RUTHES; GOULARTE, 2013). Os queijos frescos artesanais são mais frequentemente associados à contaminação por micro-organismos, visto que na maioria das vezes são fabricados utilizando leite cru e não passam por processo de maturação, ou seja, alterações físicas, bioquímicas e microbiológicas que mudam a composição química do queijo (TOZZO et al., 2015).

A contaminação do queijo adquire destaque tanto no setor industrial quanto na saúde pública, devido às perdas econômicas e ao risco da proliferação de doenças trans- 
mitidas por esse alimento (TOZZO et al., 2015), relacionados especialmente a presença de bactérias patogênicas como Escherichia coli, Salmonella sp. e Sthaphylococcus spp. (DORES et al., 2013).

Considerando o exposto, a presente pesquisa objetivou abordar a conservação de queijo com óleos essenciais extraídos de plantas, com a perspectiva de que novos estudos sejam realizados a fim de identificar compostos que tenham características que se adéquem a inclusão em um sistema de conservação de alimentos que venham a ser utilizados na conservação do queijo. Portanto, é importante conhecer métodos de controle de micro-organismos patogênicos de interesse na área de alimentos, em queijos, utilizando óleos essenciais vegetais.

\section{REFERENCIAL TEÓRICO}

\section{Variedades de queijos}

O queijo é definido como uma concentração láctea composta por proteínas, lipídios, carboidratos, sais minerais e vitaminas (OLIVEIRA et al., 2012), sendo derivado tradicional do leite, apresenta boas qualidades organolépticas e nutritivas, que faz terem grande aceitação no mercado. Os queijos são produzidos em muitas variedades quanto ao tipo, sabor, cor, forma e aroma (CORRÊA, 2014).

Segundo a Portaria de $n^{\circ} 146 / 1996$, através do Regulamento Técnico de Identidade e Qualidade dos Produtos Lácteos do Ministério da Agricultura, Pecuária e Abastecimento (MAPA), os queijos podem ser classificados de acordo com o conteúdo de matéria gorda no extrato seco, em percentagem, como: extra gordo ou duplo creme (mínimo de 60\%), gordos (entre 45,0 e $59,9 \%$ ), semi-gordo (entre 25,0 e $44,9 \%$ ), magros (entre 10,0 e 24,9\%), desnatados (menos de 10,0\%). Em relação ao conteúdo de umidade, em percentagem, os queijos classificam-se em: queijos de baixa umidade (até 35,9\%), conhecidos como queijos de massa dura, queijos de média umidade (entre 36,0 e $45,9 \%$ ), queijos de massa semi-dura, queijos de alta umidade (entre 46,0 e $54,9 \%$ ), aqueles de massa branda ou "macios", queijos de muito alta umidade (não inferior a 55,0\%), de massa branda ou "mole". Os queijos de muito alta umidade quando submetidos ou não a tratamento térmico logo após a fermentação, podem ser classificados em: queijos de muito alta umidade tratados termicamente e queijos de muito alta umidade (BRASIL, 1996).

Segundo Okura e Moacir (2010), para fabricação dos produtos lácteos, a matéria prima deve apresentar condições higiênico-sanitárias satisfatórias, pois os componentes do leite são excelentes meios para o desenvolvimento microbiano. O queijo, por ser um produto derivado do leite, apresenta elevado valor nutritivo, justificando assim sua presença na alimentação diária da maior parte da população do país, sendo dessa forma um provável vínculo de transmissão microbiana (OKURA; MOACIR, 2010).

\section{Micro-organismos associados à conta- minação do queijo}

A contaminação do queijo pode ocorrer a partir de fatores como matéria-prima de má qualidade, manipulação do produto de forma imprópria e armazenamento inadequado (VIEIRA et al., 2008). Alguns micro-organismos, tais como Listeria monocytogenes, Salmonella sp., Escherichia coli e Staphylococcus aureus, estão associados a surtos de toxinfecções alimentares envolvendo o consumo de leite e seus derivados (SANTOS, 2010).

\section{Listeria monocytogenes}

L. monocytogenes é um micro-organismo 
patógeno Gram-positivo de origem alimentar, facultativamente intracelular (PAGADALA et al., 2012). Pode causar infecções no estômago e intestino de indivíduos saudáveis, que pode levar a uma listeriose, doença que provoca graves infecções localizadas e generalizadas em humanos e em outros vertebrados, como aves e mamíferos (CLARK et al., 2010).

A L. monocytogenes é uma ameaça significativa à saúde e segurança alimentar causando preocupação em todo o mundo, principalmente em relação à contaminação de alimentos prontos para consumo, pois podem persistir nesses alimentos depois do processamento e crescer em temperaturas de refrigeração (TODD; NOTERMANS, 2011).

Segundo McCollum et al. (2013), em 2011 nos Estados Unidos esse microorganismo causou um dos mais graves surtos de doenças transmitidas por alimentos, sendo responsáveis por náuseas em 147 indivíduos, desses constatou-se 33 óbitos. A maioria das infecções causadas por alimentos tem sido associada a queijos de pasta mole (ROBERT; LEGGIADRO, 2014), leite cru e peixe (PESAVENTO et al., 2010).

Estudos mostram a incidência de $L$. monocytogenes como contaminantes de queijos em geral (BARANCELLI et al., 2014). Rosa et al. (2018), estudando a avaliação de L. monocytogenes em queijo frescal comercializado na região noroeste do estado de Paraná, observou que 55\% das amostras analisadas tiveram a presença de Listeria monocytogenes. Em trabalho com a ocorrência de Listeria monocytogenes em ricotas e queijos frescais produzidos no estado do Paraná, observou-se que $4,7 \%$ dos queijos e ricotas apresentaram-se contaminados por L. monocytogenes (BERNARDI, 2014). Em outro estudo observando presença de L. monocytogenes em queijos de pasta mole da região a Sul do Tejo, também se detectou a prevalência desse micro-organismo (GONÇALVES et al., 2017).
A alta prevalência de contaminação por essa bactéria em queijos deve-se ao fato de parte deles serem consumidos crus e a refrigeração não impedir o desenvolvimento deste micro-organismo (BARANCELLI et al., 2011).

\section{Salmonella spp.}

A Salmonella spp. é uma bactéria pertencente à família Enterobacteriaceae, Gram-negativa, não esporulada e anaeróbia facultativa (TORTORA et al., 2012). Microorganismo patógeno e responsável por graves infecções de origem alimentar, tendo grande importância para saúde pública (GONZALESBARRON et al., 2012).

Essa bactéria e responsável por gastrenterites, que provocam diarreia bacteriana. Avalia-se que de cerca de 1,8 milhões de casos de doenças diarreicas são atribuídos, na sua maioria, a Shigella spp. e Salmonella spp. (SHAO et al., 2011) e em 2012 na União Europeia foram diagnosticados mais de 90.000 casos de salmonelose (EFSA; ECDC, 2014). Em quase todos os países os surtos de infecção de origem alimentar mais comuns, estão associados à Salmonella spp. (LAURI et al., 2011).

Um dos principais veículos para a transmissão de Salmonella spp. são os alimentos de origem animal, carne de vaca, peixes, laticínios, bem como leite e queijos oriundos de leite não pasteurizado e principalmente ovos e aves (SHINOHARA et al., 2008).

Souza et al. (2017) estudando a qualidade microbiológica de queijo Minas Frescal comercializado na Zona da Mata Mineira, sendo estudado seis municípios e 50 amostras, observaram que em $40 \%$ delas foi confirmada a presença de Salmonella spp. Em trabalho realizado com pesquisa de Salmonella spp. em queijos de Coalho comercializados em feiras livres de Garanhuns, PE, pôde-se observar a presença de Salmonella spp. em $66,7 \%$ 
(8/12) das amostras de queijo de Coalho analisadas. A alta ocorrência de Salmonella spp. evidencia a falta de boas práticas de fabricação do produto, as condições precárias de higiene, armazenamento e falta de refrigeração na comercialização, que culminam no comprometimento da qualidade microbiológica (CLAUDINO, 2018).

\section{Escherichia coli}

E. coli é um micro-organismo Gramnegativo, anaeróbio facultativo, pertencente a família Enterobacteriaceae, faz parte da microbiota intestinal normal do homem e de animais de sangue quente, porém tem uma tendência a se modificar e tornase patógeno oportunista (TRABULSI; ALTHERTUM, 2008). Sua presença nos alimentos e na água indica contaminação de origem fecal. Essa bactéria pode causar infecções do trato urinário, e certas linhagens produzem enterotoxinas que causam a diarreia do viajante e ocasionalmente causam várias doenças graves de origem alimentar (TORTORA et al., 2012).

Pesquisa realizada com queijos artesanais como veículo de contaminação por $E$. coli mostrou que a $E$. coli estava presente em $50 \%$ das amostras de queijo de Coalho e em 7\% no queijo de Manteiga, comercializados em Cruz das Almas - BA (EVANGELISTA-BARRETO et al., 2016). Cardoso e Marin (2014), mostraram em trabalho com E. coli provenientes de queijo Muçarela artesanal produzido no brasil, que $(64,4 \%)$ das amostras apresentaram-se contaminadas por E. coli.

\section{Staphylococcus aureus}

$S$. aureus é um agente patógeno de seres humanos, bactéria Gram-positiva que causa doença a nível mundial (FRANÇOIS et al., 2010). Dos micro-organismos pertencentes ao gênero Staphylococcus, essa espécie é mais prevalente em surtos de intoxicação alimentar, pois a mesma apresenta grande capacidade de produzir enterotoxinas, as quais, quando ingeridas juntamente com o alimento, podem causar alguns sintomas como dores abdominais náuseas, diarreia e vômitos (JOHLER et al., 2015).

A intoxicação alimentar causada por Staphylococcus é um fator frequente de gastrenterite, entre os anos de 2008 e 2010 foram observados na China um total de 371 surtos, envolvendo 20.062 indivíduos e levando a 41 mortes (MAO et al., 2010). Os alimentos que estão mais frequentemente ligados à infecção por Staphylococcus são os produtos à base de carne, produtos de aves e ovos, alimentos cobertos e recheados com creme, chocolate, recheios de sanduíches, atum, frango, batata, macarrão, maionese, leite e produtos lácteos (FDA, 2012). Segundo Sousa et al. (2014) o uso de leite cru na produção do queijo Coalho é a principal causa de contaminação advinda da mastite bovina, onde o $S$. aureus e o principal agente etiológico.

Trabalho realizado por Souza et al. (2015) com estirpes de $S$. aureus isoladas de queijo Minas artesanal de Araxá mostrou que $93,3 \%$ dos queijos analisados tiveram a presença de $S$. aureus. S. aureus em queijos de Coalho artesanais produzidos em São Rafael, $\mathrm{RN}$, esse trabalho mostrou que todos os queijos estudados evidenciaram condições de higiene inadequadas, apresentando contaminação elevada por $S$. aureus (PEREIRA et al., 2017). Em estudo realizado com 159 amostras de queijo Muçarela para detecção de $S$. aureus, apenas 3,2\% apresentaram contaminação (GASPAROTTO et al., 2019).

\section{Conservantes}

Os conservantes são substâncias químicas, naturais ou sintéticas, adicionadas aos alimentos e a outros produtos com a finalidade de aumentar seu tempo de vida 
útil, protegendo-os de micro-organismos ou reações químicas que possam impossibilitar seu uso. Alguns conservantes foram aprovados pela legislação brasileira com o objetivo de minimizar o crescimento de patógenos em alimentos. De acordo com a Portaria de $\mathrm{n}^{\circ} 146$ de 1996 do Ministério da Agricultura, Pecuária e Abastecimento, os conservantes permitidos na fabricação de queijo são ácido sórbico, nitrato de sódio e potássio, natamicina, nisina e lisozima (BRASIL, 1996) (Tabela 1). Quanto maior a concentração utilizada, mais tempo será inibido o crescimento microbiano (DOSSIÊ, 2011). Porém, mesmo possuindo toxicidade baixa, esses aditivos podem causar efeitos tóxicos ao organismo, quando se apresentam em altas concentrações nos alimentos (FERRAND et al., 2000).

$\mathrm{O}$ ácido sórbico é utilizado na maioria dos alimentos em concentrações entre $0,05 \mathrm{e}$ $0,3 \%$. Para a inibição do crescimento de microorganismos em queijo ralado, é admitido o uso dos conservantes natamicina e ácido sórbico. O ácido sórbico é comumente utilizado, sendo permitido no produto concentrações de até $1000 \mathrm{mg} / \mathrm{kg}$ (DOSSIÊ, 2011).

O nitrato é um sal muito utilizado como aditivo na indústria alimentícia para impedir ou retardar ações de micro-organismos, protegendo o alimento da deterioração e aumentando sua vida de prateleira. $\mathrm{O}$ nitrato não é capaz de impedir a germinação dos esporos, no entanto evita o crescimento dos esporos germinados, inibindo dessa forma a multiplicação das células vegetativas. Entretanto, esse aditivo pode causar efeitos adversos quando utilizado em longo prazo, como efeitos carcinógenos, teratogênicos e mutagênicos (TERRA et al., 2004).

Natamicina é um antibiótico produzido pelo micro-organismo Streptomyces natalensis e tem ação fungicida relevante, mas ineficaz contra bactérias. É praticamente insolúvel em água, sua baixa solubilidade em água garante que esta permaneça na superfície e não migre para a matriz alimentar (CHEN et al., 2008). Os bolores são suscetíveis a concentrações mínimas inibitórias de 0,1 a $10 \mu \mathrm{g} / \mathrm{mL}$ de

Tabela 1 - Conservantes permitidos para serem utilizados na fabricação de queijos

\section{Conservantes}

Ácido sórbico e seus sais

Nitrato de sódio ou potássio

Natamicina

Nisina

$12,5 \mathrm{mg} / \mathrm{kg}$ de queijo

$1 \mathrm{mg} / \mathrm{dm}^{2}$ (superfície), $5 \mathrm{~g} / \mathrm{kg}$ não detectável a $2 \mathrm{~mm}$ de profundidade
Tipos de queijos em que é permitida utilização

\section{para utilização em queijo}

$1000 \mathrm{mg} / \mathrm{kg}$ de queijo

mau, au, mu, bu*
$50 \mathrm{mg} / \mathrm{kg}$ de queijo
$\mathrm{mu}, \mathrm{bu}$

mau, au, mu, bu

Lisozima

$25 \mathrm{mg} / \mathrm{L}$ de leite

mau, au, mu, bu

$\mathrm{mu}, \mathrm{bu}$

Fonte: Brasil (1996)

* mau: queijos de muito alta umidade; au: queijos de alta umidade; mu: queijos de média umidade; bu: queijos de baixa umidade. 
natamicina, já as leveduras são inibidas de 1 a $5 \mu \mathrm{g} / \mathrm{mL}$ de concentração, realizando ligação irreversível ao ergosterol celular do fungo levando à formação de canais iônicos e aumentando a permeabilidade celular e resultando em morte microbiana (RESA et al., 2014). A natamicina atua na superfície de queijos e não interfere no crescimento de Penicillium roquefort no interior do queijo, o que é desejável (OLIVEIRA et al., 2006).

A nisina é produzida por algumas cepas de Lactococcus lactis, sendo polipeptídeo proteico mais estável em condições ácidas, solúvel em condições aquosa e instável em soluções alcalinas e calor. Tem sido bastante utilizada em produtos alimentares, isoladamente ou em combinação com outros compostos. Apresenta atividade contra vários patógenos alimentares, como Alicyclobacillus spp., L. monocytogenes, Bacillus spp., Clostridium spp., Pediococcus spp., S. aureus, Enterococcus spp., Streptococcus haemolyticus, Lactobacillus spp., (LEE; PAIK, 2016). A nisina é a bacteriocina mais usada e aprovada pelo FDA (Food and Drug Administration) como conservante de alimentos. Foi usada pela primeira vez como conservante em queijos, e nos últimos anos começou a ser usada como conservante, em vários produtos lácteos, carnes e derivados. Não tem potencial para inibir o crescimento de microrganismos Gram-negativos e fungos, mas tem alto potencial frente a muitas bactérias Gram-positivas (SUGANTHI et al., 2012).

A lisozima é uma enzima presente nos ovos e no leite de mamíferos, comercialmente disponível e pode ser usada contra uma ampla gama de organismos de deterioração de alimentos para prolongar a vida útil de vários produtos alimentícios, incluindo carnes cruas e processadas, leite e derivados frutas e legumes (TIWARI et al., 2009). A lisozima apresenta atividade antimicrobiana, especialmente contra bactérias Gram-positivas como $L$. monocytoneges, no entanto, não tem efeito significativo nas bactérias Gram-negativas (RAWDKUEN et al., 2012).

\section{Plantas com potencial antimicrobiano}

Compostos de origem vegetal, em especial os condimentos e seus derivados (óleos essenciais), exibem potencial relevante como agentes antimicrobianos, apresentando nova perspectiva de utilização na conservação de alimentos (TAJKARIMI et al., 2010). Alguns dos condimentos que se destacam por apresentarem forte atividade antimicrobiana são cravo-da-índia, orégano e alecrim. Os óleos essenciais destas especiarias possuem compostos químicos, tais como carvacrol, cinamaldeído e eugenol, tidos como os principais componentes químicos responsáveis pela atividade antimicrobiana (ZAWIRSKAWOJTASIAK; WASOWICZ, 2009).

\section{Rosmarinus officinalis L. (Alecrim- pimenta)}

Rosmarinus officinalis L. é uma erva aromática pertencente à família Lamiaceae (BEGUM et al., 2013). Utilizada geralmente como agente aromatizante de alimentos e, medicinalmente, apresenta propriedades antiinflamatórias, diuréticas e antimicrobianas (ARRANZ et al., 2015; TEIXEIRA et al., 2013) sendo aplicado no tratamento de câncer e diabetes (AFONSO et al., 2013).

Estudos mostram a atividade antibacteriana do óleo essencial e do extrato de $R$. officinalis frente a diferentes microorganismos, tais como, E. coli e $S$. aureus (WITKOWSKA et al., 2013; CASTANO et al., 2010), Bacillus subtilis (KLANCNIK et al., 2009), L. monocytogenes (JOSIPOVIC et al., 2015) e Salmonella (MAZZARRINO et al., 2015; RIBEIRO et al., 2012).

\section{Origanum vulgare L. (Orégano)}

Origanum vulgare é uma erva aromática pertencente à família Lamiaceae, nativa da 
Europa e Ásia, cultivada no mundo todo, utilizada na culinária e para fins medicinais (CRISTEA et al., 2008), no tratamento de problemas menstruais, distúrbios digestivos (SIKANDER et al., 2012), como fortificante e estimulante (SANKAR et al., 2013).

Pesquisas realizadas com óleo essencial de $O$. vulgare mostram que esse óleo apresenta ação antibacteriana sobre as cepas de $E$. coli, S. aureus e Salmonella spp., exibindo como componentes químicos majoritários o 4-terpineol e timol, indicando que essa planta apresenta potencial antimicrobiano natural (ARAUJO et al., 2015). Além de possuir várias outras propriedades biológicas como expectorante (ARSLAN et al., 2013), antioxidante (KARAKAYA et al., 2011), antimutagênico (GULLUCE et al., 2012) e antimicrobiano (BARANAUSKIENE et al., 2013).

Em trabalho desenvolvido por Hernandez-Hernandez et al. (2014) com óleo essencial da folhas de orégano para determinar sua atividade antimicrobiana frente a bactérias patógenas comumente encontrados em alimentos frescos, observouse ação contra os micro-organismos Brochothrix thermosphacta, Pseudomonas fragi, Salmonella sp. e Micrococcus luteus, tendo o timol e carvacrol como os principais compostos químicos, o que lhes confere propriedades antimicrobianas (MURIELGALET et al., 2012).

\section{Syzygium aromaticum (Cravo-da- índia)}

O Syzygium aromaticum, popularmente conhecido como cravo-da-índia, é uma planta de porte arbóreo com copa alongada, pertence à família das mirtáceas, e bastante utilizada como condimento na culinária, por apresentar aroma e sabor marcante, devido à presença do eugenol (AFFONSO et al., 2012). Pesquisas mostram que o cravo-da-índia apresenta várias atividades farmacológicas como antiviral, antidiabética, anestésica, anti-inflamatória (AFFONSO et al., 2012), antimicrobiana (ALMEIDA et al., 2013; MOON et al., 2011), inseticida e repelente (AFFONSO et al., 2013). Almeida et al. (2013) verificaram a atividade antibacteriana do óleo essencial de cravo-da-índia frente a $S$. aureus. Silva et al. (2015) avaliando o óleo essencial da folha do $S$. aromaticum reportaram alto potencial antimicrobiano contra $E$. coli e $S$. aureus.

O óleo de cravo-da-índia testado sobre o crescimento de várias bactérias Gram-negativas evidenciou forte atividade antimicrobiana frente à Escherichia coli, Pseudomonas aeruginosa, Klebsiella pneumoniae, Enterobacter aerogene, Salmonella typhi, Klebsiella ozaenae, Shigella dysentriae e Proteus mirabilis (SABAHAT; PERWEEN, 2008).

\section{Óleos essenciais utilizados na conservação de queijo}

Pesquisas vêm sendo desenvolvidas com novos agentes antimicrobianos provenientes de plantas que possam ser aplicados em produtos alimentícios (MACHADO et al., 2011). De acordo com Haida et al. (2007) as plantas usadas como condimentos possuem propriedades antimicrobianas, antioxidantes e medicinais. As características antimicrobianas dessas especiarias têm despertado interesse dos pesquisadores, devido a perspectivas de sua utilização como aditivo natural em alimentos, a fim de reduzir as infecções e intoxicação provenientes de alimentos contaminados.

Os óleos essenciais são misturas de compostos voláteis e lipossolúveis, tais como, monoterpenos, sesquiterpenos e fenilpropanóides, geralmente apresentam densidades menores do que a água e facilmente se difundem através da membrana celular, facilitando sua penetração, promovendo as interações com seu sitio de ação, possuem composição química complexa e são originados do metabolismo secundário 
das plantas, seus constituintes variam de compostos mais simples a mais complexos, como hidrocarbonetos terpênicos, alcoóis simples e terpênicos, aldeídos, cetonas, éteres, fenóis, óxidos, peróxidos, furanos, ácidos orgânicos, cumarinas (RUBIOLO et al., 2010).

Os óleos essenciais mostram efeito inibitório na integridade da membrana contra as bactérias patogênicas de origem alimentar. O vazamento intracelular de material é um fenômeno geral que resulta em morte celular. A natureza hidrofóbica dos óleos essenciais pode interferir na membrana lipídica bacteriana, resultando em aumento da permeabilidade dos constituintes celulares, o que está de acordo com outros compostos fenólicos. Bactérias Gram-positivas são geralmente mais suscetíveis que bactérias Gram-negativas. Os lipopolissacarídeos da parede celular das bactérias Gramnegativas podem criar uma barreira para as macromoléculas e compostos hidrofóbicos, impedindo que os compostos ativos nos óleos essenciais cheguem à membrana citoplasmática (HACWYDRO et al., 2017; WEN et al., 2016). Alguns estudos mostram a utilização de óleos essenciais de plantas na conservação de alguns tipos de queijos (Tabela 2).

Okura e Moacir (2010), trabalhando com a produção de queijo Minas Frescal utilizando as especiarias orégano $(O$. vulgare $)$ e salsinha (P. sativum) para inibir o crescimento de E. coli, verificaram que esta bactéria mostrou-se sensível aos antimicrobianos utilizados. De

Tabela 2 - Óleos essenciais de partes de plantas utilizados na conservação de alguns tipos de queijos e seus efeitos sobre micro-organismos patogênicos

\section{Espécie vegetal Nome comum Espécie bacteriana Queijo Referências}

\begin{tabular}{|c|c|c|c|c|}
\hline Allium sativum & Alho & S. choleraesuis & Quark & Mallet (2011) \\
\hline Origanum vulgare & Orégano & E. coli & $\begin{array}{l}\text { Minas } \\
\text { Frescal }\end{array}$ & Okura; Moacir (2010) \\
\hline Origanum vulgare & Orégano & $\begin{array}{l}\text { E. coli; } \text { S. aureus; } \\
\text { P. aeruginosa; } \\
\text { L. monocytogenes; } \\
\text { S. choleraesuis }\end{array}$ & Quark & Mallet (2011) \\
\hline Origanum vulgare & Orégano & $\begin{array}{l}\text { L. monocytogenes; } \\
\text { S. aureus }\end{array}$ & Coalho & Souza (2015) \\
\hline $\begin{array}{l}\text { Petroselinum } \\
\text { sativum }\end{array}$ & Salsinha & E. coli & $\begin{array}{l}\text { Minas } \\
\text { Frescal }\end{array}$ & Okura; Moacir (2010) \\
\hline $\begin{array}{l}\text { Rosmarinus } \\
\text { officinalis }\end{array}$ & Alecrim & E. coli & Coalho & Ribeiro (2011) \\
\hline $\begin{array}{l}\text { Schinus } \\
\text { terebinthifolius } \\
\text { Raddi }\end{array}$ & Pimenta rosa & L. monocytogenes & $\begin{array}{l}\text { Minas } \\
\text { Frescal }\end{array}$ & $\begin{array}{c}\text { Dannenberg et al. } \\
\text { (2016) }\end{array}$ \\
\hline
\end{tabular}


acordo com Mallet (2011) ao utilizar óleos essenciais de alho (A. sativum) e orégano (O. vulgare) na conservação de queijo tipo Quark, ficou evidente a inibição satisfatória do orégano frente a cepas de E. coli, S. aureus, L. monocytogenese $S$. choleraesuis, enquanto o óleo de alho inibiu apenas a cepa de $S$. choleraesuis.

Ao utilizar o óleo essencial de alecrim (R. officinalis) na conservação do queijo de Coalho sobre o crescimento de Escherichia coli, Ribeiro et al. (2011) observaram a susceptibilidade da bactéria à ação do óleo essencial de alecrim, enquanto pesquisa realizada com queijo de Coalho utilizando óleo essencial de $O$. vulgare mostrou potencial ação antibacteriana frente a $S$. aureus e $L$. monocytogenes (SOUZA, 2015).

O efeito do óleo essencial de pimenta rosa (S. terebinthifolius), utilizado sobre o crescimento de L. monocytogenes em queijo Minas Frescal, mostrou que o óleo essencial tem potencial frente ao micro-organismo estudado (DANNENBERG et al., 2016).

\section{CONSIDERAÇÕES FINAIS}

Embora os queijos sejam considerados microbiologicamente seguros, surtos de origem alimentar associados a este alimento ainda vem ocorrendo, sendo importante o uso de antimicrobianos naturais, como os óleos essenciais, para conservação de alimentos, como alternativa aos conservantes sintéticos. No entanto, é necessário realizar estudos sensoriais do produto, observar sua interação com os componentes intrínsecos do alimento e a ação sobre os patógenos associados a cada alimento, entre outros fatores, para indicar esses antimicrobianos naturais como aditivo nos alimentos.

\section{AGRADECIMENTOS}

Os autores agradecem a Coordenação de Aperfeiçoamento de Pessoal de Nível Superior - CAPES, pelas bolsas de estudo concedidas ao primeiro e segundo autor.

\section{REFERÊNCIAS}

AFFONSO, R. S. et al. Applications of molecular modeling in the design of new insect repellents targeting the odorant binding protein of Anopheles gambiae. Journal of the Brazilian Chemical Society, v. 24, n.3, p. 473-482, 2013. DOI: 10.5935/01035053.20130059

AFFONSO, R. S. et al. Aspectos químicos e biológicos do óleo essencial de cravo da índia. Revista Virtual de Química, v. 4, n. 2, p. 146-161, 2012. DOI: 10.5935/19846835.20120012

AFONSO, M. S. et al. Phenolic compounds from rosemary (Rosmarinus officinalis L.) attenuate oxidative stress and reduce blood cholesterol concentrations in diet-induced hypercholesterolemic rats. Nutrition \& Metabolism, v. 10, article n. 19, p. 1-9. 2013. DOI:10.1186/1743-7075-10-19

ALMEIDA, A. C. et al. Antimicrobial activity of essential oils of Syzygium aromaticum L. and Ocimum gratissimum L. Revista Brasileira de Ciência Veterinária, v. 20 , n. 4 , p. $248-251$, 2013. DOI: $10.1590 /$ S0074-02761999000500022

ARAUJO, L. S. et al. Composição química e susceptibilidade do óleo essencial de orégano (Origanum vulgare L.) frente a cepas de Escherichia coli, Staphylococcus aureus e Salmonella choleraesuis. Boletim do Centro de Pesquisa e Processamento de Alimentos, v. 33, n. 1, p. 73-78, 2015.

ARRANZ, E. et al. Anti-inflammatory activity of the basolateral fraction of Caco- 
2 cells exposed to a rosemary supercritical extract. Journal of Functional Foods, v. 13, p. 384-390, 2015. DOI: 10.1016/j. jff.2015.01.015

ARSLAN, U. et al. Evaluation of the use of ammonium bicarbonate and oregano (Origanum vulgare ssp. Hirtum) extract on the control of apple scab. Journal of Phytopathology, v. 161, n. 6, p. 382-388, 2013. DOI: $10.1111 /$ jph. 12082

BARANAUSKIENE, R. et al. Harvesting time influences the yield and oil composition of Origanum vulgare L. ssp. vulgare and ssp. hirtum. Industrial Crops and Products, v. 49, p. 43-51, 2013. DOI: 10.1016/j. indcrop.2013.04.024

BARANCELLI, G. V. et al. Listeria monocytogenes: ocorrência em produtos lácteos e suas implicações em saúde pública. Arquivos do Instituto Biológico, v. 78, n. 1, p. 155-168, 2011.

BARANCELLI, G. V. et al. Pulsed-Field Gel Electrophoresis of Listeria monocytogenes isolates from cheese manufacturing plants in São Paulo, Brazil. International Journal of Food Microbiology, v. 173, p. 21-29, 2014. DOI: $10.1016 /$ j.ijfoodmicro.2013.12.018

BEGUM, A. et al. An in-depth review on the medicinal flora Rosmarinus officinalis (Lamiaceae). Acta Scientiarum Polonorum Technologia Alimentaria, v. 12, n. 1, p. $61-73,2013$.

BERNARDI, G. Avaliação de métodos de deteç̧ão e ocorrência de Listeria monocytogenes em ricotas e queijos frescais produzidos no Estado do Paraná. 2014. 87 f. Dissertação (Mestrado em Ciências Farmacêuticas) - Ciências da Saúde, Universidade Federal do Paraná, Curitiba, 2014.
BRASIL. Ministério da Agricultura, do Abastecimento e da Reforma Agrária. Portaria $n^{\circ} 146$, de 7 de março de 1996. Aprova os Regulamentos Técnicos de Identidade e Qualidade dos Produtos Lácteos. Diário Oficial da República Federativa do Brasil: seção 1, Brasília, DF, n. 48, p. 3977, 11 mar. 1996.

CARDOSO, P.; MARIN, J. M. Resistência antimicrobiana de isolados de Escherichia coli provenientes de queijo Muçarela artesanal produzido no Brasil. Ars Veterinaria, v. 30, n. 2, p. 104-108, 2014. DOI: 10.15361/2175-0106.2014v30n2p104-108

CASTANO, P. H. I. et al. Bactericidal activity of ethanolic leaf extract and leaf essential oil of Rosmarinus officinalis L. on some food borne bacteria. Vitae, v. 17, n. 2, p. 149-154, 2010 .

CHEN, G. et al. Natamycin production by Streptomyces gilvosporeus based on statistical optimization. Journal of Agricultural and Food Chemistry, v. 56, n. 13, p. 5057-5061, 2008. DOI: $10.1021 /$ jf800479u

CLARK, C. G. et al. Surveillance for Listeria monocytogenes and listeriosis, 1995 - 2004. Epidemiology \& Infection, v. 138 , n. 4 , p. 559-572, 2010. DOI:10.1017 /S0950268809990914

CLAUDINO, A. R. F. Pesquisa de Salmonella spp. em queijos de Coalho comercializados em feiras livres de Garanhuns-PE. 2018. 49f. Trabalho de Conclusão de Curso (Graduação em Medicina Veterinária) - Departamento de Medicina Veterinária, Universidade Federal Rural de Pernambuco, Garanhuns, 2018.

CORRÊA, F. T. Desenvolvimento de queijo maturado com tiras de árvores cultivadas no Brasil. 2014. 81 f. Dissertação (Mestrado 
em Tecnologia do Leite e Produtos Láticos) Ciências dos Alimentos, Universidade Federal de Lavras, Lavras, 2014.

CRISTEA, T. O. et al. Considerations regarding the effects of growth regulators over the in vitro morphogenetic reaction at Origanum vulgare L. Journal of Plant Development, v. 15, p. 133-138, 2008.

DANNENBERG, G. S. et al. Antimicrobial and antioxidant activity of essential oil from pink pepper tree (Schinus terebinthifolius Raddi) in vitro and in cheese experimentally contaminated with Listeria monocytogenes. Innovative Food Science and Emerging Technologies, v. 36, p. 120-127, 2016. DOI: 10.1016/j.ifset.2016.06.009

DORES, M. T. et al. Enterotoxigenic potential of Staphylococcus aureus isolated from artisan Minas cheese from the Serra da Canastra, MG, Brazil. Food Science and Technology, v. 33, n. 2, p. 271-275, 2013. DOI: 10.1590/s010120612013005000033

DOSSIÊ Conservantes. Revista Food Ingredients Brasil, n. 18, p. 2-10, 2011.

EUROPEAN FOOD SAFETY AUTHORITY (EFSA); EUROPEAN CENTRE FOR DISEASE PREVENTION AND CONTROL (ECDC). The European Union Summary Report on trends and sources of zoonoses, zoonotic agents and food-borne outbreaks in 2012. EFSA Journal, v. 12, n. 2, 2014. DOI: $10.2903 /$ j.efsa. 2014.3547

EVANGELISTA-BARRETO, N. S. et al., Queijos artesanais como veículo de contaminação de Escherichia coli e estafilococos coagulase positiva resistentes a antimicrobianos. Revista Brasileira de Higiene e Sanidade Animal, v. 10, n. 1, p. 55-67, 2016.
FAVA, L. W. et al. Características de queijos artesanais tipo Colonial comercializados em uma feira agropecuária. Acta Scientiae Veterinariae, v. 40, n. 4, p. 1-6, 2012.

FERRAND, C. et al. Mutagenicity and genotoxicity of ascorbic acid-amine reaction products. Food Additives and Contaminants, v. 17, n. 11, p. 895-901, 2000. DOI: $10.1080 / 026520300750038063$

FOOD AND DRUG ADMINISTRATION (FDA). Bad Bug Book: Foodborne Pathogenic Microorganisms and Natural Toxins Handbook. 2. ed. 2012. Disponível em: https://wayback. archive-it.org/7993/20170405001300/ https://www.fda.gov/downloads/Food/ FoodborneIllnessContaminants/UCM297627. pdf

FORSYTHE, S. J. Microbiologia da Segurança Alimentar. 2. ed. Porto Alegre: Artmed, 2013. 602 p.

FRANÇOIS, P. et al. Proteomic approaches to study Staphylococcus aureus pathogenesis. Journal of Proteomics, v. 73, n. 4, p. 701-708, 2010. DOI: $10.1016 /$ j.jprot.2009.10.007

GASPAROTTO, P. H. G. et al. Avaliação microbiológica para detecção de Staphylococcus aureus em queijos tipo Muçarela. Revista Ciência e Saúde Animal, v. 1, n. 1, p. 16-25, 2019. DOI: 10.6084/ m9.figshare.11312459.v2

GONÇALVES, M. et al. Presença de Listeria monocytogenes em queijos de pasta mole da região a sul do Tejo. Portuguese Journal of Public Health, v. 35, p. 37-43, 2017. DOI: $10.1159 / 000477648$

GONZALES-BARRON, U. A. et al. A risk characterization model of Salmonella typhimurium in Irish fresh pork sausages. 
Food Research International, v. 45, n. 2, p. 1184-1193, 2012. DOI: $10.1016 /$ j. foodres. 2010.04 .036

GULLUCE, M. et al. Isolation of some active compounds from Origanum vulgare L. ssp. vulgare and determination of their genotoxic potentials. Food Chemistry, v. 130 , n. 2 , p. $248-253$, 2012. DOI: $10.1016 /$ j. foodchem.2011.07.024

HACWYDRO, K. et al. Essential oils as food eco-preservatives: Model system studies on the effect of temperature on limonene antibacterial activity. Food Chemistry, v. 235 , p. $127-135$, 2017. DOI: $10.1016 /$ j. foodchem.2017.05.051

HAIDA, K. S. et al. Avaliação in vitro da atividade antimicrobiana de oito espécies de plantas medicinais. Arquivos de Ciências da Saúde da UNIPAR, v. 11, n. 3, p. 185-192, 2007. DOI: 10.25110/arqsaude. v11i3.2007.2037

H E R N A N D E Z - H E R N A N D E Z, E. et al. Microencapsulation, chemical characterization and antimicrobial activity of mexican (Lippia graveolens H.B.K.) and european (Origanum vulgare L.) oregano essential oils. The Scientific World Journal, v. 2014, p. 1-12, 2014. DOI: $10.1155 / 2014 / 641814$

JOHLER, S. et al. Further evidence for staphylococcal food poisoning outbreaks caused by egc-encoded enterotoxins. Toxins, v. 7, n. 3, p. $997-1004$, 2015. DOI: 10.3390/ toxins 7030997

JOSIPOVIC, R. et al. Improved properties and microbiological safety of novel cottage cheese containing spices. Food Technology and Biotechnology, v. 53, n. 4, p. 454-462, 2015. DOI: $10.17113 / \mathrm{ftb} .53 .04 .15 .4029$
KARAKAYA, S. et al. Antioxidant and antimicrobial activities of essential oils obtained from oregano (Origanum vulgare ssp hirtum) by using different extraction methods. Journal of Medicinal Food, v. 14, n. 6, p. 645-652, 2011. DOI: 10.1089/jmf.2010.0098

KLANCNIK, A. et al. In vitro antimicrobial and antioxidant activity of commercial rosemary extract formulations. Journal of Food Protection, v. 72, n. 8, p. 1744-1752, 2009. DOI: $10.4315 / 0362-028 x-72.8 .1744$

LAURI, A. et al. Comprehensive analysis of Salmonella sequence polymorphisms and development of a LDR-UA assay for the detection and characterization of selected serotypes. Applied Microbiology and Biotechnology, v. 91, n. 1, p. 189-210, 2011. DOI: $10.1007 / \mathrm{s} 00253-011-3308-1$

LEE, N.; PAIK, H. Status, antimicrobial mechanism, and regulation of natural preservatives in livestock food systems. Journal of Food Science, v. 36, n. 4, p. $547-557,2016$. DOI: $10.5851 /$ kosfa.2016.36.4.547

MACHADO, T. F.; BORGES, M. F.; BRUNO, L. M. Documentos 145: Aplicação de antimicrobianos naturais na conservação de alimentos. Fortaleza: Embrapa Agroindústria Tropical, 2011.

MALLET, A. C. T. Utilização de óleos essenciais de condimentos na conservação de queijo tipo quark. 2011. 131f. Tese (Doutorado em Ciências dos Alimentos) - Faculdade de Ciências de Alimentos, Universidade Federal de Lavras, Lavras, 2011.

MAO, X. et al. Epidemiological characteristics of bacterial foodborne disease during the year 2003-2007 in China. Chinese Journal of Food Hygiene, v. 22, p. 224-228, 2010. 
MAZZARRINO, G. et al. Salmonella enterica and Listeria monocytogenes inactivation dynamics after treatment with selected essential oils. Food Control, v. 50, n. 10, p. 794-803, 2015. DOI: 10.1016/j. foodcont.2014.10.029

McCOLLUM, J. T. et al. Multistate outbreak of listeriosis associated with cantaloupe. New England Journal Medicine, v. 369, n. 10, p. 944-953, 2013.

MOON, S. E. et al. Synergistic effect between clove oil and its major compounds and antibiotics against oral bacteria. Archives of Oral Biology, v. 56, n. 9, p. 907-916, 2011. DOI: 10.1016/j.archoralbio.2011.02.005

MURIEL-GALET, V. et al. Development of antimicrobial films for microbiological control of packaged salad. International Journal of Food Microbiology, v. 157, n. 2, p. 195-201, 2012. DOI: 10.1016/j. ijfoodmicro.2012.05.002

OKURA, M. H.; MOACIR, J. Avaliação das condições higiênico-sanitárias de queijos Minas Frescal produzidos com leite cru, leite pasteurizado e de queijo temperado em alguns municípios da região do triangulo mineiro. Revista do Instituto de Laticínios Cândido Tostes, v. 65, p. 33-42, 2010.

OLIVEIRA, L. M. A. et al. Avaliação da qualidade de queijos ralados para proteção da saúde pública. Revista do Instituto Laticínio Candido Tostes, v. 67, n. 384, p. 41-47, 2012.

OLIVEIRA, T. M. et al. Avaliação do teor de natamicina em queijos Gorgonzola através de quantificação espectrofotométrica. Revista do Instituto de Laticínio Cândido Tostes, v. 61, n. 349, p. 48-50, 2006.

PAGADALA, S. et al. Prevalence, characterization and sources of Listeria monocytogenes in blue crab (Callinectus sapidus) meat and blue crab processing plants. Food Microbiology, v. 31, n. 2, p. 263-270, 2012. DOI: $10.1016 /$ j.fm.2012.03.015

PEREIRA. T. M. F. et al. Staphylococcus aureus e Salmonella sp. em queijos de Coalho artesanais produzidos em São Rafael, Rio Grande do Norte. Revista Verde de Agroecologia e Desenvolvimento Sustentável, v. 12, n. 2, p. 358-361, 2017. DOI: $10.18378 /$ rvads.v12i2.4829

PESAVENTO, G. et al. Prevalence and antibiotic susceptibility of Listeria spp. isolated from raw meat and retail foods. Food Control, v. 21, n. 5, p. 708-713, 2010. DOI: 10.1016/j.foodcont.2009.10.012

RAWDKUEN, S. et al. Antimicrobial activity of some potential active compounds against food spoilage microorganisms. African Journal Biotechnology, v. 11, p. 13914-13921, 2012. DOI: 10.5897/AJB12.1400

RESA, C. P. O. et al. Natamycin efficiency for controlling yeast growth in models systems and on cheese surfaces. Food Control, v. 35, n. 1, p. 101-108, 2014. DOI: 10.1016/j.foodcont.2013.06.049

RIBEIRO, D. R. Avaliação da atividade antimicrobiana do óleo essencial de alecrim (Rosmarinu sofficinalis L.) frente a bactérias isoladas de alimentos: estudos in vitro e em matriz alimentícia. 2011. 103f. Dissertação (Mestrado em Ciência de Alimentos) Faculdade de Farmácia, Universidade Federal da Bahia, Salvador, 2011.

RIBEIRO, D. S. et al. Avaliação do óleo essencial de alecrim (Rosmarinus officinalis L.) como modulador da resistência bacteriana. Ciências Agrárias, v. 33, n. 2, p. 687-696, 2012. 
DOI: $10.5433 / 1679-0359.2012 v 33 n 2 p 687$

ROBERT, J.; LEGGIADRO, M. D. Vital signs: Listeria illnesses, deaths, and outbreaks - United States, 2009-2011.Pediatric Infectious Disease Journal, v. 33, n. 3, p. 271, 2014.

ROSA, G. et al. Evaluation of Listeria monocytogenes in Frescal cheese traded in the northwestern region of the state of Paraná. Jornal Interdisciplinar de Biociências, v. 3, n. 1, 2018.

RUBIOLO, P. et al. Essential oils and volatiles: sample preparation and analysis. A review. Flavour Fragance Journal, v. 25, n. 1, p. 282-290, 2010. DOI: 10.1002/ffj.1984

RUTHES, L. D.; GOULARTE, M. M. M. Qualidade microbiológica de queijos de diversas regiões do Estado do Paraná. Higiene Alimentar, v. 27, n. 218/219, p. 172-176, 2013.

SABAHAT, S.; PERWEEN T. In vitro antibacterial activity of clove against Gramnegative bacteria. Pakistan Journal of Botany, v. 40, n. 5, p. 2157-2160, 2008.

SANKAR, R. et al. Origanum vulgare mediated biosynthesis of silver nanoparticles for its antibacterial and anticancer activity. Colloids and Surfaces B: Biointerfaces, v. 108 , n. 1 , p. $80-84$, 2013. DOI: $10.1016 /$ j. colsurfb.2013.02.033

\section{SANTOS, J. C. Avaliação do efeito} antimicrobiano de óleos essenciais sobre micro-organismos patogênicos em vôngole (Anomalocardia brasiliana). 2010. 83f. Dissertação (Pós-Graduação em Ciência de Alimentos) - Faculdade de Farmácia, Universidade Federal da Bahia, Salvador, 2010 .
SHAO, Y. et al. Development of multiplex loop-mediated isothermal amplificationRFLP (mLAMP-RFLP) to detect Salmonella spp. and Shigella spp. in milk. International Journal of Food Microbiology, v. 148, n. 2, p. 75-79, 2011. DOI: $10.1016 / \mathrm{j}$. ijfoodmicro.2011.05.004

SHINOHARA, N. K. S. et al. Samonella spp., importante agente patógeno veiculado em alimentos. Revista Ciências \& Saúde Coletiva, v. 13, n. 5, p. 1675-1683, 2008. DOI:10.1590/s1413-81232008000500031

SIKANDER, M. et al. Hepatoprotective effect of Origanum vulgare in Wistar rats against carbon tetrachloride-induced hepatotoxicity. Protoplasma, v. 250, n. 2, p. 483-493, 2012. DOI: $10.1007 / \mathrm{s} 00709-012-0431-5$

SILVA, A. A. et al. Avaliação da atividade antimicrobiana dos óleos essenciais de Thymus vulgaris (tomilho), Syzygium aromaticum (cravo-da-índia) e Rosmarinus officinalis (alecrim) e dos conservantes benzoato de sódio e sorbato de potássio em Escherichia coli e Staphylococcus aureus. Boletim do Centro de Pesquisa e Processamento de Alimentos, v. 33, n. 1, p. 111-117, 2015. DOI: 10.5380/cep.v33i1.43814

SOUSA, A. Z. B. et al. Aspectos físicoquímicos e microbiológicos do queijo tipo Coalho comercializados em estados do Nordeste do Brasil. Arquivo Instituto Biológico, v. 81, n. 1, p. 30-35, 2014. DOI: 10.1590/s1808-16572014000100006

SOUZA, G. T. Efeitos do óleo essencial de Origanum vulgare $\mathrm{L}$. sobre o crescimento de bactérias patogênicas e tecnológicas em queijo de Coalho. 2015. 70f. Dissertação (Mestrado em Ciências e Tecnologia de Alimentos) Centro de Tecnologia, Universidade Federal de Paraíba, João Pessoa, 2015. 
SOUZA, I. A. et al. Qualidade microbiológica de queijo Minas frescal comercializado na Zona da Mata Mineira. Revista do Instituto de Laticínios Cândido Tostes, v. 72, n. 3, p. 152-162, 2017.

SOUZA, V. et al. Estirpes de Staphylococcus aureus isoladas de queijo Minas artesanal de Araxá. Ars Veterinaria, v. 31, n. 1, p. 19-23, 2015. DOI: $10.15361 / 2175-0106.2015 v 31 n$ 1p19-23

SUGANTHI, V. et al. Lantibiotic nisin: natural preservative from Lactococcus lactis. International Research Journal Pharmacy, v. 3, p. 13-19, 2012.

TAJKARIMI, M. M. et al. Antimicrobial herb and spice compounds in food. Food Control, v. 21, n. 9, p. 1199-1218, 2010. DOI: 10.1016/j.foodcont.2010.02.003

TEIXEIRA, B. et al. Chemical composition and antibacterial and antioxidant properties of commercial essential oils. Industrial Crops and Products, v. 43, n. 0, p. 587-595, 2013. DOI: $10.1016 /$ j.indcrop.2012.07.069

TERRA, A. B. M.; FRIES, L. L.; TERRA, N. N. Particularidades na Fabricação de Salames. 1. ed. São Paulo: Editora Varela, 2004.

TIWARI, B. K. et al. Application of natural antimicrobials for food preservation. Journal of Agricultural and Food Chemistry, v. 57, p. 5987-6000, 2009. DOI: 10.1021/jf900668n

TODD, E. C. D.; NOTERMANS, S. Surveillance of listeriosis and its causative pathogen, Listeria monocytogenes. Food
Control, v. 22, n. 9, p. 1484-1490, 2011. DOI: 10.1016/j.foodcont.2010.07.021

TORTORA, G. J. et al. Microbiologia.10. ed. Porto Alegre: Artmed, 2012. 894 p.

TOZZO, K. et al. Avaliação microbiológica de queijos coloniais da região de Cascavel - PR. Higiene Alimentar, v. 29, n. 244-245, p. 149-154, 2015.

TRABUlsi, L. R.; Althertum, F. Microbiologia. 5. ed. São Paulo: Atheneu, 2008. 533 p.

VIEIRA, K. P. et al. Contaminação de queijo Minas Frescal por bactérias patogênicas: Um risco à saúde. Revista ConScientiae Saúde, v. 7 , n. 2 , p. 201-206, 2008. DOI: $10.5585 /$ conssaude.v7i2.685

WEN, P. et al. Encapsulation of cinnamon essential oil in electrospun nanofibrous film for active food packaging. Food Control, v. 59, p. $366-376,2016$. DOI: $10.1016 / \mathrm{j}$. foodcont.2015.06.005

WITKOWSKA, A. M. et al. Evaluation of antimicrobial activities of commercial herb and spice extracts against selected foodborne bacteria. Journal of Food Research, v. 2, n. 37-54, 2013. DOI: 10.5539/jfr. v2n4p37

Z A W I R S K A - W O J T A S I A K , R.; WASOWICZ, E.G. C. Analysis of rosemary aroma isolate traditionally by distillation and by SPME. Journal of Essential Oil Research, v. 21, n. 1, p. 8-15, 2009. DOI: 10.1080/10412905.2009.9700094 